\title{
Vascular function, insulin resistance and fatty acids
}

\author{
H. O. Steinberg ${ }^{1}$, A. D. Baron ${ }^{2}$ \\ ${ }^{1}$ Indiana University School of Medicine, Indianapolis, Indiana, USA \\ ${ }^{2}$ Amylin Pharmaceuticals, San Diego, California, USA
}

\begin{abstract}
Over the past 10 years it has become clear that intact vascular function, especially at the level of the endothelium, is paramount in the prevention or delay of cardiovascular disease. It has also become clear that insulin itself, in addition to its metabolic actions, directly effects vascular endothelium and smooth muscle. Insulin, at normal physiologic concentrations, causes changes in skeletal muscle blood flow in healthy, insulin-sensitive subjects. Insulin's effect on the endothelium is mediated through its own receptor and insulin signalling pathways, resulting in the increased release of nitric oxide. Insulin's vascular actions are impaired in insulin-resistant conditions such as obesity, Type II (non-insulin-dependent) diabetes mellitus
\end{abstract}

and hypertension, which could contribute to the excessive rates of cardiovascular disease in these groups. Insulin-resistant states of obesity and Type II diabetes show a multitude of metabolic abnormalities that could cause vascular dysfunction. Non-esterified fatty acid levels increase long before hyperglycaemia becomes present. Raised non-esterified fatty acids impair insulin's effect on glucose uptake in skeletal muscle and the vascular endothelium and thus could have detrimental effects on the vasculature, leading to premature cardiovascular disease. [Diabetologia (2002) 45:623-634]

Keywords Fatty acids, insulin, metabolism, blood flow, endothelial, nitric oxide.
Insulin's effects are associated with energy metabolism, largely involving the regulation of glucose and fatty acid metabolism. Indeed, insulin plays a vital role in the regulation of glucose and fatty acid homeostasis and an important role in amino acid metabolism. As such, it facilitates the storage of fuels and macromolecules in liver, muscle and fat. Over the last 15 years, it has become clear that insulin's action is not

Received: 21 May 2001 / Revised: 28 November 2001

Published online: 4 April 2002

C) Springer-Verlag 2002

Coresponding author: A. D. Baron, Amylin Pharmaceuticals, San Diego, CA 92121, USA, e-mail: abaron@amylin.com Abbreviations: NO, Nitric oxide; EDNO, endothelium derived nitric oxide; L-NMMA, L-n-mono-methyl-l-arginine; Mch, methacholine chloride; SNP, sodium nitroprusside; LBF, leg blood flow; IRS, insulin receptor substrate; P13K, phosphatidyl-inositol 3 kinase restricted to these classic target tissues. Insulin receptors have been described in the brain and in beta cells of the pancreas where insulin appears to play a role in energy metabolism and insulin secretion, respectively $[1,2,3,4]$. In addition, insulin receptors are found in endothelial and vascular smooth muscle cells and insulin has been shown to modulate vascular tone and tissue blood flow [5].

Insulin-resistant states such as obesity, Type II (non-insulin-dependent) diabetes mellitus and polycystic ovarian syndrome [7] are characterized by impairment of insulin's classic actions to stimulate glucose uptake in skeletal muscle and fat cells, and inhibit lipolysis in adipose tissue resulting in increased systemic free fatty acid concentrations $[6,7]$. The mechanisms involved in insulin resistance are not fully understood but are probably multifactorial. The effects of insulin resistance on classic insulin actions have been described at length but the effects of insulin re- 

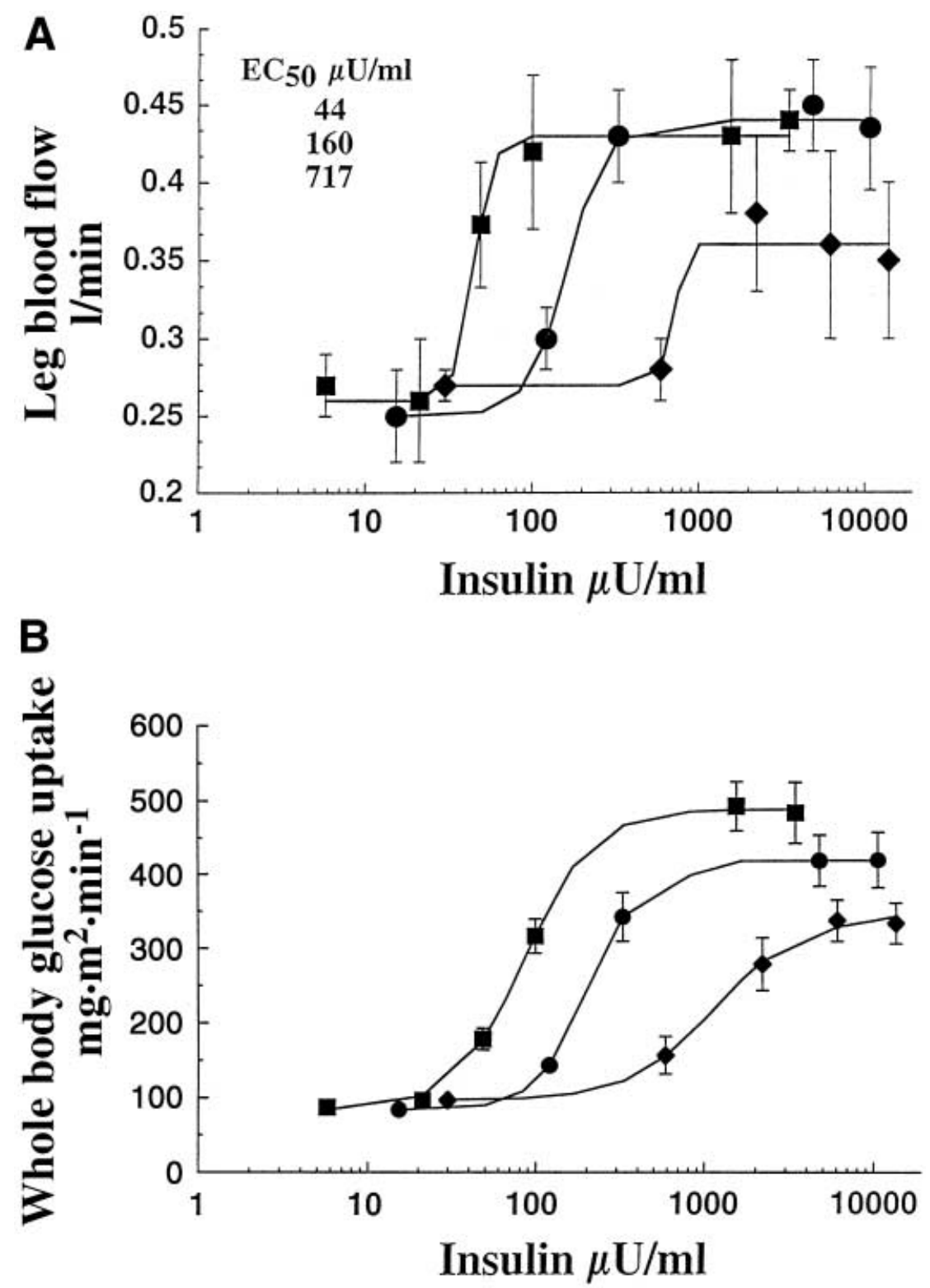

Fig. 1. A Rates of leg blood flow in response to graded hyperinsulinaemic euglycaemic clamp in lean $(\boldsymbol{\square})$, obese $(\mathbf{O})$ and Type II diabetic (NIDDM $\bullet$ ) subjects. B Rates of whole body glucose uptake $\left(\mathrm{mg} \cdot \mathrm{m}^{2} \mathrm{~min}\right)^{1}$ in response to graded hyperinsulinaemic euglycaemic clamp in lean $(\boldsymbol{\square})$, obese $(\boldsymbol{)})$ and Type II diabetic (NIDDM $\diamond)$ subjects

sistance on non-classical effector systems are less well known.

While insulin resistance has received a great deal of attention with respect to its role in the pathogenesis of Type II diabetes, it is now clear that insulin resistance independent of hyperglycaemia is associated with a twofold to threefold increase in the risk of cardiovascular mortality, with only $50 \%$ of the excess mortality being explained by classic cardiovascular risk factors such as blood pressure and cholesterol increases [8]. In this review, we give evidence suggest- ing that impairment of insulin action at the level of the vasculature contributes to the more accelerated and more severe atherosclerotic process observed in clinical states of insulin resistance $[9,10]$. The purpose of this review is to summarize the current understanding of vascular endothelial function in relation to insulin sensitivity with emphasis on the role of fatty acids on insulin action and vascular reactivity.

\section{Insulin and the vasculature}

The first evidence demonstrating that insulin has vasoactivity independent of its glucose lowering action was obtained in anaesthetized dogs [11]. However, it took another 10 years before it was recognized that insulin had a direct effect on the vasculature in humans. In 1989, Laakso et al. demonstrated a dosedependent effect of insulin to increase leg blood flow in healthy insulin sensitive subjects [12]. Although 


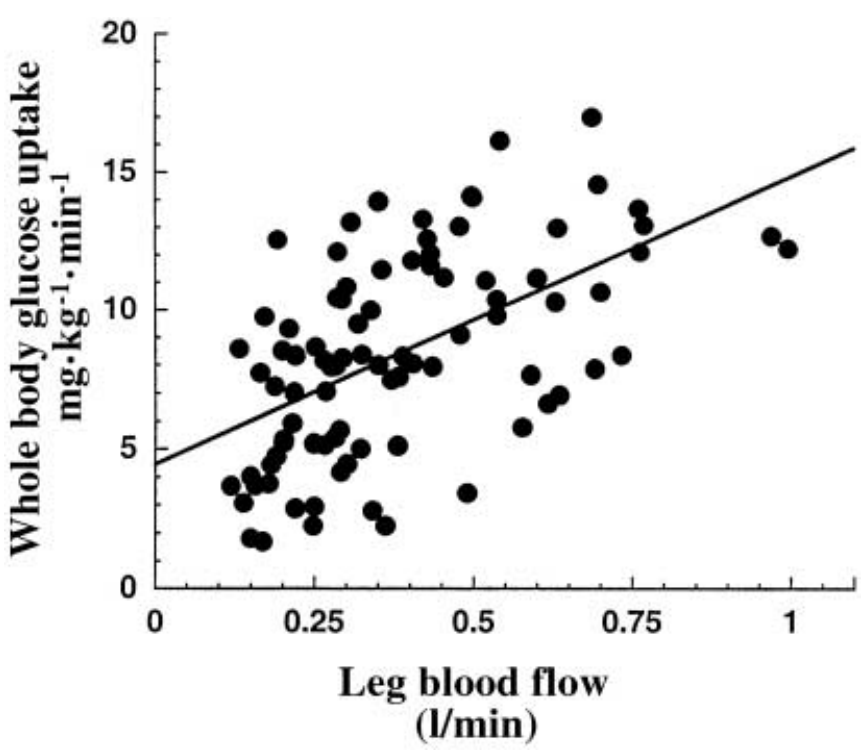

Fig. 2. Relation between rates of leg blood flow (1/min) and rates of whole body glucose uptake in response to euglycaemic hyperinsulinaemia $(r=0.56, p=0.001, n=88)$

some groups were able to reproduce the findings, others were not able to observe insulin's effect on augmenting blood flow or only after prolonged infusions $[13,14,15,16]$. Subsequent studies showed that hyperinsulinaemia can increase cardiac output without changing systemic blood pressure while simultaneously increasing blood flow to the leg [17]. According to Ohm's law $(\mathrm{R}=\mathrm{P} / \mathrm{F})$, this increment in blood flow $(\mathrm{F})$ without a change in blood pressure $(\mathrm{P})$ indicates that vascular resistance $(\mathrm{R})$ in skeletal muscle must fall. In other words, it appears that insulin preferentially dilates skeletal muscle vessels and as such redirects the increment in cardiac output to insulin sensitive tissues. Figure 1A illustrates the effect of physiological insulin concentrations which cause an approximately twofold increase in LBF above baseline in lean insulin sensitive individuals. These studies were done during euglycaemic clamp conditions and so it follows that this insulin effect is independent of changes in glycaemia. The magnitude of vasodilation appears related not only to the insulin concentration but also the rate of insulin-stimulated glucose metabolism [18]. Figure 2 illustrates the high degree of correlation between the rate of insulin-stimulated glucose uptake and the extent of vasodilation.

In a separate study, we simultaneously determined the time course of insulin-mediated vasodilation and insulin's effect on tissue glucose extraction [19]. Glucose extraction is assessed by obtaining the arteriovenous glucose differences in response to euglycaemic hyperinsulinaemia and largely reflects the changes in tissue permeability to glucose. During saline control studies, the T1/2 (the time to reach the half-maximal effect) for insulin-mediated vasodilation was $32 \pm$ 16 min comparable to the $\mathrm{T} 1 / 2$ for glucose extraction which was $36 \pm 4$ min in lean healthy subjects. The fact that T1/2 for insulin-mediated vasodilation and glucose extraction were similar suggests that both effects are important determinants of rates of glucose uptake and thus insulin sensitivity. The fact that vasodilation is temporally related to glucose extraction and metabolism and that the magnitude of vasodilation is related to the absolute rate of whole body glucose metabolism, suggests that glucose metabolism and vasodilation are tightly coupled processes. In the same study [19], prevention of insulin-mediated vasodilation by direct intra-arterial infusion of an inhibitor of nitric oxide synthase, L-NMMA, reduced glucose uptake by about $35 \%$ indicating that changes in blood flow modulate glucose uptake. Using different vasoconstrictor drugs such as angiotensin II [20], norepinephrine [21], or ouabain [22], similar reductions in insulin-mediated glucose uptake have been shown supporting the notion that changes in blood flow can modulate insulinmediated glucose uptake. On the other hand, data from studies evaluating the effect of pharmacologically-increased blood flow on insulin-mediated glucose uptake are less clear. Our group demonstrated that methacholine-induced doubling of leg blood flow increased leg glucose uptake by $40 \%$ beyond that achieved by hyperinsulinaemia alone [23]. However, increases in blood flow were not observed to augment insulin-mediated glucose uptake in several studies using the vasodilator sodium nitroprusside [24], adenosine [25], or bradykinin [26]. More recently, elegant studies by Clark and co-workers [27, 28, 29, 30] have indicated that capillary recruitment in response to insulin is more important than bulk flow to determine rates of tissue insulin-mediated glucose uptake. Thus, while the role of insulin to modulate glucose metabolism by its vascular effects has been controversial [31, $32]$, it has been generally accepted that insulin causes vasodilation through the release of NO from the vascular endothelium.

\section{Mechanism of insulin-mediated vasodilation}

Viewed simply, insulin-mediated vasodilation could be due to a direct effect of insulin on blood vessels or mediated indirectly, through modulation of sympathetic nervous system or by a mechanism linked to the rate of skeletal muscle metabolism, or a combination of these.

The endothelium is a diaphanous cellular monolayer lining the lumen of the vasculature throughout the body. This large organ has been recognized for its barrier and transport functions and was once thought to be mostly passive in these capacities. This view changed after the intact endothelium was found to be required for the relaxation of arterial smooth muscle by acetylcholine [23]. Other investigators subsequently demonstrated that nitric oxide (NO) release in re- 


\section{Hypothetical model of insulin / edno interaction}

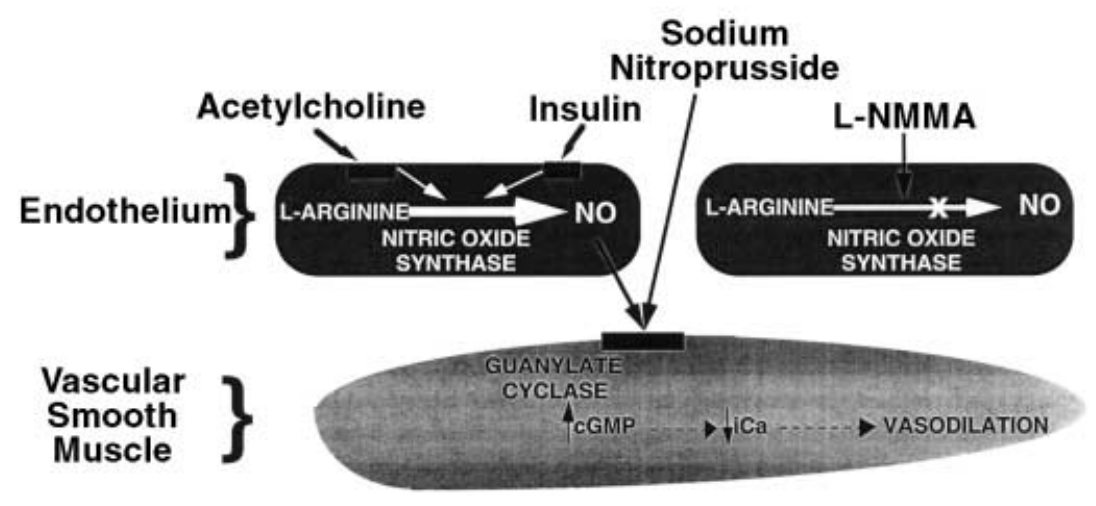

Fig. 3. Simplified model showing endothelial and vascular smooth muscle cell interaction and pharmacological tools to assess different aspects of this interaction in vivo

sponse to muscarinergic agonists such as acetylcholine chiefly accounts for the endothelium-dependent relaxing activity [34]. Endothelium-derived NO is now widely recognized as the most potent endogenous vasodilating substance.

Endothelium-derived NO is a gas that is synthesized from the precursor L-arginine in a reaction catalysed by nitric oxide synthase and continuously released from the endothelium. NO released from the endothelium diffuses through the subendothelial space to the vascular smooth muscle where it binds to the heme group of guanylate cyclase and stimulates the generation of cyclic GMP (cGMP) which in turn leads to a reduction in intracellular $\mathrm{Ca}++$ resulting in smooth muscle relaxation and vasodilation [35].

One can assess endothelium-dependent vasodilation in vivo as shown in Figure 3. This can be accomplished by measuring blood flow increments (or change in vascular resistance) in response to agents that cause the release of NO from the endothelium such as the muscarinergic agonists acetylcholine or by measuring blood flow reductions in response to inhibitors of NO synthase such as the arginine analogue L-n-monomethyl-1-arginine (L-NMMA) [36]. The blood flow response to L-NMMA thus provides an assessment of NO-dependent vascular tone. To differentiate between endothelium-dependent and endothelium-independent vasodilation, NO donor compounds such as sodium nitroprusside (SNP) can be utilized to directly activate cGMP in vascular smooth muscle that ultimately results in vascular smooth muscle relaxation and vasodilation. Thus, enhanced or blunted NO action at the level of the vascular smooth muscle can be determined by measuring blood flow increases in response to SNP. Together, these pharmacological manoeuvres allow the assessment of endothelial-dependent and endothelial-inde- pendent vasodilation as well as NO-dependent vascular tone in humans.

In the early 1990's, our group and others tested the hypothesis that insulin causes vasodilation by the EDNO dependent mechanism [14, 37]. To this end, L-NMMA, a specific inhibitor of NO synthase, was administered directly into the femoral artery while measuring the LBF response during a systemic infusion of either saline or insulin plus glucose (euglycaemic hyperinsulinaemic clamp) designed to approximately double LBF. In response to L-NMMA, LBF decreased by about $20 \%$ during saline alone, suggesting that about $20 \%$ of basal vascular tone is NO-dependent. Importantly, we showed that during insulin infusion nearly $40 \%$ of vascular tone was dependent on NO. L-NMMA completely cancelled the insulin-induced vasodilation suggesting that insulinmediated vasodilation is entirely NO-dependent. However, because this effect of L-NMMA infusion was observed after $4 \mathrm{~h}$ of hyperinsulinaemia, insulin could vasodilate primarily by an NO-independent mechanism and NO could be only secondarily released as a result of increased shear stress. To explore this possibility, we did another study in which LBF responses to hyperinsulinaemia were measured during either intra-arterial infusion of saline or L-NMMA over a period of $90 \mathrm{~min}$. L-NMMA was infused at the onset of the insulin infusion, changes in LBF in response to hyperinsulinaemia were completely cancelled, suggesting that insulin causes vasodilation via the release of NO (Fig. 4) [19]. A number of studies by a variety of techniques have now confirmed the NO dependency of insulin-mediated vasodilation in other vascular beds such as the forearm [13, 38, 39], supporting the notion that this effect is probably generalised in insulin sensitive tissues. These studies confirm that NO plays a role in insulin mediated vasodilation but does not distinguish between increased rates of production or release or both or increased NO action (sensitivity or responsiveness) in the vascular smooth muscle.

To determine whether insulin increases NO synthesis and release or NO action, responses to graded in- 


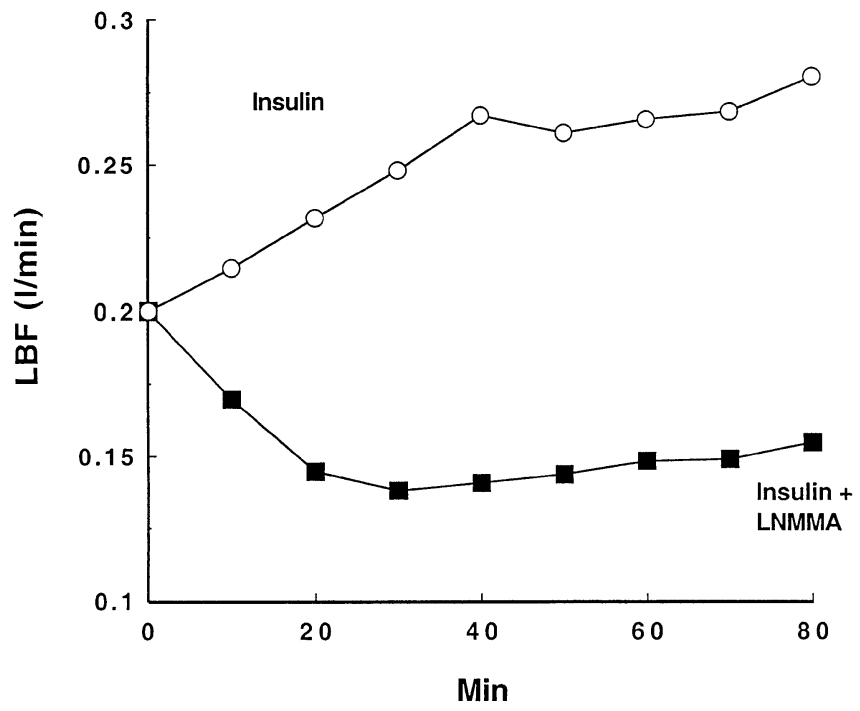

Fig. 4. Leg blood flow (LBF) in response to systemic infusion of insulin alone (euglycaemic hyperinsulinaemia) and with superimposed intra-femoral artery infusion of L-NMMA

tra-femoral infusions of the endothelium-dependent vasodilator methacholine chloride $(\mathrm{MCh})$ and the endothelium-independent vasodilator sodium nitroprusside (SNP) were studied in normal subjects during infusion of saline or hyperinsulinaemic euglycaemia [37]. LBF increased in response to MCh by nearly $50 \%$ during hyperinsulinaemia compared to saline control. In contrast, hyperinsulinaemia had no effect on endothelium-independent vasodilation, as measured by the response to SNP. These observations suggest that insulin's effect of vasodilating skeletal muscle vasculature is not mediated by enhanced NO action on the vascular smooth muscle but depends on increased production or release of NO or both.

Although most studies examining insulin's effects on the vasculature agree that insulin-mediated vasodilation is achieved by increments in NO production or release or both, it is still possible that factors other than hyperinsulinaemia are required to induce vasodilation, as studies were able to detect blood flow increases in response to systemic hyperinsulinaemia but were not able to reproduce these observations during a direct intra-arterial infusion of insulin. One mechanism for the discrepant findings in the forearm between systemic and local insulin administration could be vasodilation in response to intra-arterial hyperinsulinaemia only after blockade of the endothelin A receptor [40]. Investigators propose that insulin's direct vasodilator effect in the forearm is masked by concomitant rise in endothelin levels. However, their observation that insulin does not increase blood flow during local intra-arterial insulin administration in the forearm is in contrast to our observation in the leg [37]. We demonstrated increases in LBF in response to infusions of insulin directly in the femoral artery. These discrepant observations could be due to differ- ences in the behaviour of the skeletal muscle examined, ie leg compared with forearm, or due to differences in the sensitivity of the blood flow measurement methods, plethysmography vs. thermodilution [32].

\section{In vitro studies}

Together, these in vivo studies indicate that insulin vasodilates skeletal muscle vasculature by a net release of endothelium derived NO (EDNO). Insulin's effect of increasing vasodilation has also been observed in isolated organ bath vascular strip preparations [41] where it increased endothelium-dependent vascular smooth muscle relaxation. Furthermore, insulin appears to blunt the responses to a number of vasoconstrictor agents [42, 43, 44]. However, in vivo studies and vascular preparations are not able to define whether the NO release represents a direct insulin effect at the level of the endothelial cell or whether it is mediated indirectly. In a study of NO production in response to insulin in primary cultures of human umbilical vein endothelial cells (HUVEC) insulin was found to cause a dose-dependent, saturable increase in the production of NO [45]. This insulin-stimulated production of NO was completely blocked by pre-incubation of cells with L-NAME (an inhibitor of eNOS). Importantly, they demonstrated that pre-incubation with genistein (a tyrosine kinase inhibitor) also completely blocked the production of $\mathrm{NO}$ in response to insulin indicating that the insulin signalling mechanism to effect NO release was mediated by a tyrosine kinase, most probably the insulin receptor itself.

More direct evidence that the insulin receptor tyrosine kinase is necessary to mediate the effect of insulin on production of $\mathrm{NO}$ has been obtained using HUVEC that were transfected with either wild-type insulin receptors or kinase-deficient mutant insulin receptors [46]. Overexpression of wild-type insulin receptors leads to a threefold increase in NO produced in response to maximal insulin stimulation while cells overexpressing kinase-deficient insulin receptors respond like untransfected control cells.

After insulin binds to its receptor and subsequent activation of the insulin receptor tyrosine kinase subunit, two major pathways are turned on. The first is the phosphorylation of cellular substrates such as IRS-1 and IRS-2, which then activates phosphatidyl 3-kinase (PI3K) pathway. Activation of PI3K is necessary for glucose transport in skeletal muscle. Wortmannin, an inhibitor of PI3K, blocks the production of NO in response to insulin in HUVEC. This indicates that PI3K is necessary for insulin-induced NO production in the endothelial cell. Furthermore, other groups have reported that the serine/threonine protein kinase $\mathrm{Akt} / \mathrm{PKB}$, part of the classic insulin signalling pathway involved in activation of glucose transport, phosphorylates and activates eNOS leading to in- 


\section{Shared signaling between insulin stimulated glucose transport and NO production}

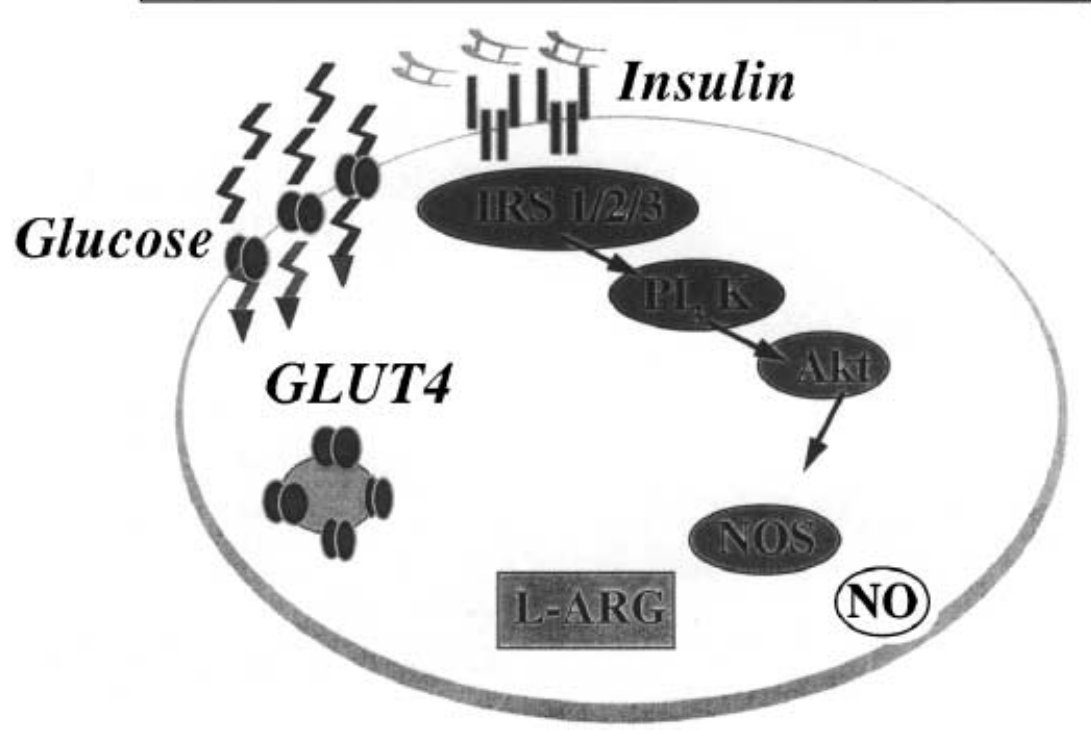

Fig. 5. Hypothetical signalling pathway connecting insulin receptor activation with translocation of Glut 4 and nitric oxide (NO) release

creased NO production $[47,48]$. Thus, eNOS is an Akt substrate linking signal transduction by Akt to the release of NO (Fig. 5). In contrast to the PI3K insulin signalling pathway, the mitogen-activated protein kinase pathway which is thought to result in VSMC growth and migration and in regulation of PAI-1 production, does not participate in insulin-mediated NO release.

Insulin does not only acutely increase NO production through the PI3K pathway but also induces eNOS gene expression [49]. Endothelial cells exposed to physiologic concentrations of insulin for $2-8 \mathrm{~h}$ increased e-NOS mRNA, protein and activity suggesting that insulin could chronically modulate NO production and vascular tone and reactivity.

\section{Insulin resistance and vascular function}

So far, we have established that (i) in lean normal subjects, insulin vasodilates in a dose-dependent fashion; (ii) this insulin-mediated vasodilation is dependent on NO released from the endothelial cells. Moreover, we have previously established that (iii) changes in tissue perfusion modulate rates of glucose uptake under conditions of euglycaemic hyperinsulinaemia [23]. Glucose uptake in skeletal muscle is decreased in insulinresistant states such as obesity and Type II diabetes. Because rates of glucose uptake are determined at least in part by tissue perfusion, we investigated whether insulin-mediated vasodilation is impaired in obesity and Type II diabetes. In other words, we examined whether insulin resistance was associated with blunted increments in tissue perfusion rates in response to euglycaemic hyperinsulinaemia.

The results of a series of studies have indicated that insulin-resistant subjects with Type II diabetes had severely impaired increments in LBF [50]. Even at extremely high prevailing insulin concentrations, LBF increased by only $40 \%$ which was less than half the increment observed in the lean, more insulin-sensitive control subjects (Fig. 1A). Obese subjects showed a LBF response to the graded insulin administration that was intermediate [12]. Obese subjects were able to mount the same maximum LBF response to insulin as subjects. However, to achieve the same LBF response, much higher insulin concentrations were required indicating that obese subjects had resistance to insulin's vasodilating action. Interestingly, the dose-response curves for insulin-mediated glucose intake (Fig. 1B) in subjects of varying insulin sensitivity (effect on glucose uptake) largely parallel those for the dose response curves for insulin's action to vasodilate (Fig. 1A). That is to say the most insulin-resistant subjects display the greatest impairment in insulin-mediated vasodilation, suggesting a strong association between insulin-mediated modulation of vascular tone and glucose metabolism. However, the notion that insulin-mediated glucose uptake and vasodilation could be associated is not accepted by all [31].

Although the above findings strongly suggest that obesity and Type II diabetes have decreased NO release, this conclusion is only inferential. To determine whether insulin-mediated NO release is impaired in insulin resistance, we measured the effects of maximally effective doses of insulin to stimulate femoral venous 
flux of the stable oxidative products of $\mathrm{NO}, \mathrm{NO} 2$ and $\mathrm{NO} 3(\mathrm{NO} 2+\mathrm{NO} 3=\mathrm{NOx})$ in subjects with a wide range of insulin sensitivity. NOx flux equals the product of LBF and NOx (NOx flux=NOx*LBF). Basal NOx flux rates during saline infusion were not different between subject groups showing a wide range of rates of insulin-mediated whole body glucose uptake (insulin sensitivity) [51]. In contrast, during maximal insulin stimulation, athletes (the most insulin sensitive group) exhibited a twofold increase in NOx flux above baseline, while patients with Type II diabetes showed no change in NOx flux above basal. Lean healthy subjects and obese subjects showed increases in NOx flux which were intermediate between those achieved by the most insulin-sensitive (athletes) and the most insulin-resistant (diabetes) subjects with lean subjects mounting a stronger NOx flux increment than the obese subjects. More importantly, changes in NOx flux were proportional to rates of whole-body glucose uptake. Thus, the data are consistent with the notion that insulin-mediated NO production occurs as a function of insulin sensitivity with insulin-resistant subjects showing reduced insulin-stimulated NO production.

Endothelial dysfunction in the insulin-resistant obese subjects and patients with Type II diabetic subjects might not be limited to the response to insulin but be more generalised. This notion is important because cardiovascular risk has been shown to relate to coronary endothelial dysfunction [52, 53, 54], which in turn is correlated to endothelial dysfunction in the peripheral vasculature [55]. Thus, endothelial dysfunction in obesity and Type II diabetes could explain some of the excess mortality in these insulin-resistant states [56, 57, 58, 59] which cannot be accounted for by classic risk factors such as age, cholesterol concentrations, blood pressure and smoking etc [8].

Therefore, to further test the notion that obesity and Type II diabetes are associated with endothelial dysfunction which is not limited to the response to insulin, we measured leg blood flow (LBF) responses to graded intra-femoral artery infusions of methacholine chloride (MCh) and sodium nitroprusside (SNP) in groups of normal lean controls, and subjects with uncomplicated obesity and Type II diabetes (Fig. 6A). The results of these studies indicated that in males, obesity was associated with impaired response to the endothelium-dependent vasodilator MCh [60]. In contrast, the response to the endothelium-independent vasodilator SNP was not different between healthy obese and lean males. Importantly, obese patient with Type II diabetes did not show further reductions in the response to MCh over obese non-diabetic males indicating that in males the effect of obesity in itself is sufficient to induce maximal vascular endothelial dysfunction. Again, the response to SNP was not different between lean normal, obese, and patients with diabetes regardless of gender. Interestingly, we discovered differences in the response to MCh between females and
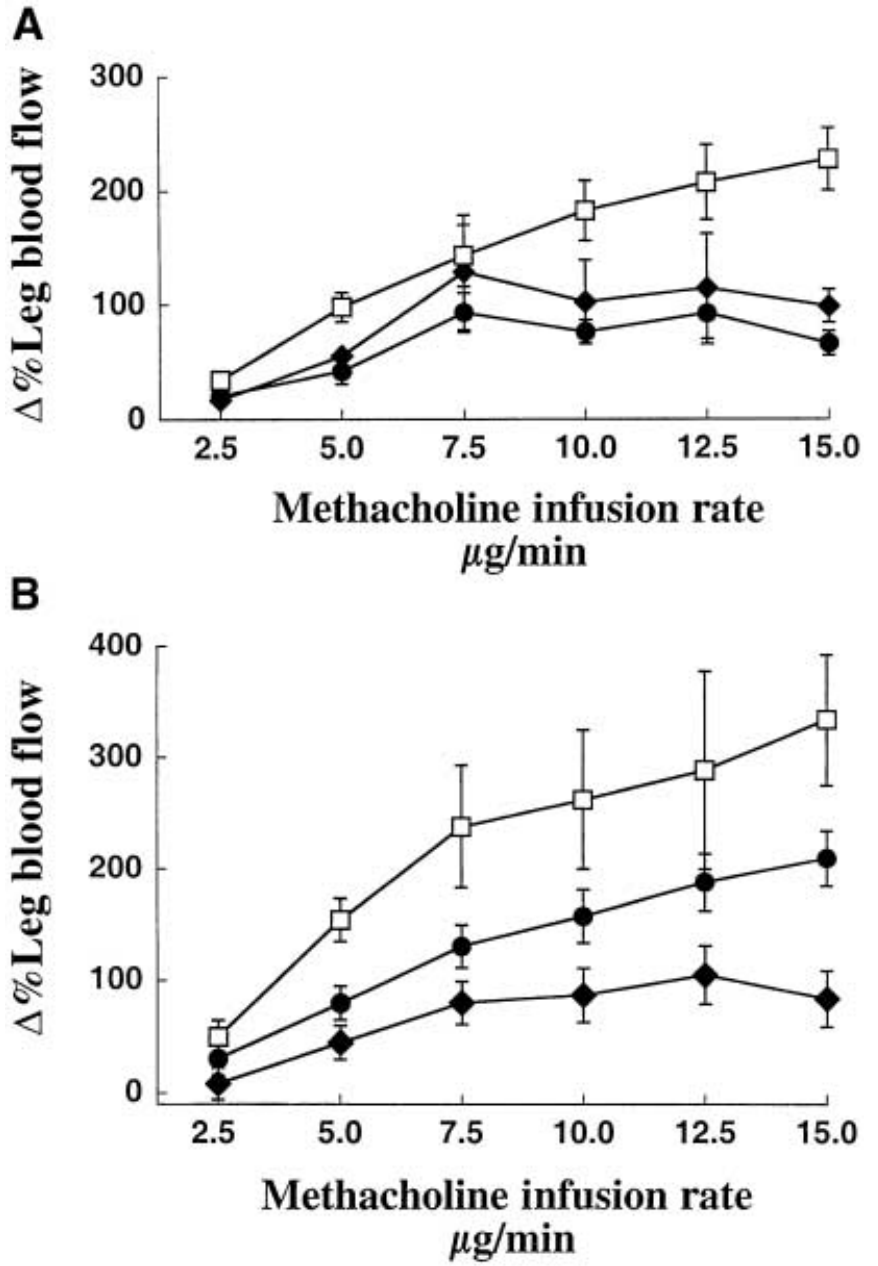

Fig. 6. A Changes in leg blood flow $(\Delta \%)$ in response to graded intra-femoral artery infusions of the endothelium-dependent vasodilator methacholine chloride in lean normal $(-\square-)$, obese $(--)$, and Type II diabetic male subjects $(-\bullet-)$. B Changes in leg blood flow $(\Delta \%)$ in response to graded intra-femoral artery infusions of the endothelium-dependent vasodilator methacholine chloride in lean normal $(-\square-)$, obese (--) and Type II diabetic female subjects $(-\bullet-)$

males. Compared to male counterparts, lean and obese females showed greater increments in LBF in response to MCh [60]. Nevertheless, obesity in the women was also associated with impaired endothelial function. Interestingly, in obese women, diabetes was associated with further deterioration of endothelial function resulting in $\mathrm{LBF}$ responses to MCh (Fig. 6B) comparable to those in obese and diabetic males [60]. These data indicate that premenopausal females have higher NO production in response to MCh than males and that this gender difference is cancelled by Type II diabetes. The mechanism(s) by which obesity and Type II diabetes affect vascular function are not clear, but given the overlap in insulin signal transduction mechanisms between skeletal muscle and endothelial cells, it is possible that whatever reduces insulin sensitivity in muscle also does so in the endothelium. In other words, insulin resistance could interfere with the 
signalling pathways leading to NO production affecting the same signalling molecules shared by both endothelial and skeletal muscle cells.

One other potential explanation for the combination of impaired insulin signalling pathways in skeletal muscle and endothelial cells might be that this represents the parallel manifestations of a shared more proximal disorder. This shared disorder would be then responsible for the parallel development of both insulin resistance and vascular dysfunction as suggested by the fetal insulin hypothesis for example [61].

The notion that endothelial function itself is impaired in insulin-resistant states such as obesity and Type II diabetes is further supported by data obtained in Zucker rats demonstrating that in aorta and microvessels of these animals, there was decreased phosphorylation of insulin receptor substrate 1 and 2, and decreased PI3K and Akt activation [62]. Basal e-NOS expression was reduced, and insulin's ability to increase e-NOS expression was blunted [49]. Other rat models of insulin resistance such as the JCR-La-cp rat have been reported to exhibit vascular dysfunction [63]. Furthermore, in vivo experiments applying the euglycaemic hyperinsulinaemic clamp technique also revealed reduced activation of the PI3K pathway but intact insulin signalling through the mitogen-activated signalling pathway [64], suggesting that obesity and Type II diabetes induce selective insulin resistance in the PI3K signalling pathway in vascular tissue.

Taken together, simple obesity and insulin resistance are associated with marked endothelial dysfunction. This endothelial dysfunction seems to be independent of other variables such as cholesterol, age or blood pressure that are known to modulate endothelial function. Insulin resistance induces alterations in insulin signalling within the vascular wall, which could account for reduced NO production. In turn, reduced NO action could be instrumental in accelerating the atherosclerotic disease process.

\section{Effects of non-esterified fatty acids on the vasculature and insulin sensitivity}

The mechanism(s) responsible for endothelial dysfunction in insulin-resistant states are not well understood. One of the characteristic metabolic abnormalities of insulin-resistant states is higher circulating non-esterified fatty acid (NEFA) concentrations. Importantly, under insulin-resistant conditions, NEFA concentrations are not only higher under fasting conditions but also fail to suppress appropriately in response to insulin in the postprandial state [65]. Thus, the skeletal muscle and the vasculature of insulin-resistant patients are constantly exposed to higher NEFA concentrations.

A number of groups have shown that higher circulating NEFA concentrations lead to resistance to insulin-mediated glucose uptake $[66,67,68]$. The time
Effect of low dose NEFA $(\sim 1500 \mu \mathrm{mol})$ on endothelium dependent vasodilation

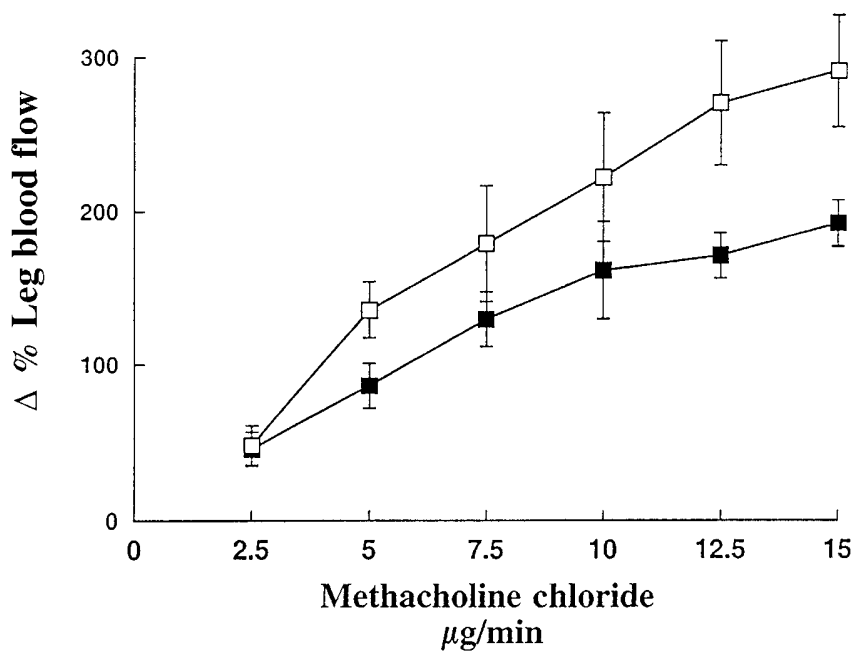

Fig. 7. Changes in leg blood flow $(\Delta \%)$ in response to graded intra-femoral artery infusions of the endothelium-dependent vasodilator methacholine chloride before $(-\square-)$ and after $2 \mathrm{~h}$ of raised non-esterified fatty acid (

course for higher NEFA concentrations to induce insulin resistance appears somewhat variable but lies between 3.5 and 8 h $[69,70]$. Higher NEFA concentration is associated with blunted insulin-stimulated increases in intracellular glucose-6-phosphate and lower intracellular glucose concentrations resulting in reduced rates of glycogen storage and glucose oxidation [67]. The mechanism by which increases in NEFA concentrations reduce skeletal muscle glucose metabolism is not well understood. NEFA did not change insulin receptor autophosphorylation indicating that the first step in the insulin signalling pathway remains intact [68] and that NEFA increases possibly impaired other more downstream effects in the insulin signalling cascade. PI3K activity increased nearly fourfold in response to hyperinsulinaemia [71]. Importantly, higher FFA concentrations for $5 \mathrm{~h}$ blunted the effect of insulin to activate PI3K by $90 \%$. Thus, the data indicate that NEFA increases interfere with insulin signalling in PI3K in rat skeletal muscle. Because intact PI3K signalling is required for insulin's effect to release endothelial NO, together these results suggest that higher amounts of NEFA could be, at least in part, responsible for the vascular abnormalities observed in clinical states of insulin resistance.

To test the hypothesis that NEFA increases impair endothelial function, we measured LBF responses to graded intra-femoral artery infusions of $\mathrm{MCh}$ in groups of lean normal subjects [72]. Under basal conditions, LBF increased by more than $291 \pm 36 \%$ in response to MCh. Subsequently, increases in NEFA concentrations were achieved by systemic administration of Intralipid $20 \%$ at $45 \mathrm{cc} / \mathrm{h}$ and heparin sulfate $(0.1 \mathrm{mU} \cdot \mathrm{kg} \cdot \mathrm{min})$. After 2-h raised NEFA concentrations, the graded intra-arterial infusions of MCh were 
repeated (Fig. 7). Short-term 2-h increases of NEFA concentrations resulted in blunting of the LBF response to MCh. With NEFA concentrations elevated twofold to threefold above baseline concentrations, the maximal LBF response to $\mathrm{MCh}$ was only $193 \pm 15 \%$. Moreover, two-to threefold increases of NEFA achieved by suppression of endogenous insulin secretion using somatostatin (increased lipolysis) resulted in a comparable impairment of endothelial function. The ability of somatostatin to induce endothelial dysfunction was cancelled by simultaneous administration of a low dose insulin $\left(4 \mathrm{mU} \cdot \mathrm{m}^{2} \mathrm{~min}\right) \mathrm{de}-$ signed to replace basal insulin concentrations and normalize circulating NEFA concentrations. This systemic low dose insulin infusion replaced basal insulin concentrations and resulted in normalization of NEFA concentrations. In contrast to this NEFA effect on endothelium-dependent vasodilation, the LBF response to the endothelium-independent vasodilator SNP remained unchanged. These data suggest that NEFA increases, achieved by increased endogenous lipolysis or by administration of exogenous fat substrate, impairs endothelial NO release and production but not NO action. In support of our observations a similar effect of NEFA increase on forearm blood flow responses to acetylcholine was reported [73]. Other supporting evidence that NEFA increases impair vascular function comes from the effect of FFA on changes of brachial artery diameter in response to postischaemic hyperaemia [74]. Clearly, brachial artery diameter increased to a lesser extent after NEFA increases compared to basal conditions. Taken together, these data indicate that NEFA increases in themselves cause endothelial dysfunction.

To assess whether NEFA increases reduced basal NO-dependent vascular tone, the LBF response to the NO synthase inhibitor L-NMMA was measured either under basal conditions or after $2 \mathrm{~h}$ of NEFA increases in lean healthy volunteers [70]. The fall in LBF in response to L-NMMA is believed to represent the NO-dependent component of vascular tone. In healthy control subjects, LBF fell by about $18 \%$ in response to L-NMMA $(16 \mathrm{mg} / \mathrm{min})$, whereas after $2 \mathrm{~h}$ of raised NEFA, LBF decreased by only $9 \%$ in response to the same dose of L-NMMA ( $p<0.05$ between groups). These data indicate that short-term NEFA increase is sufficient to impair basal (shear stress induced) and stimulated (MCh induced) NO production.

The mechanism(s) by which raised NEFA impairs (i) basal NO-dependent blood flow; (ii) response to $\mathrm{MCh}$; and (iii) postischaemic hyperaemia is not well characterized. Raised NEFA could cause vascular endothelial dysfunction either indirectly by increased release of vasoconstrictor substances such as endothelin 1 or through a direct effect on the endothelial NO system or both. Raised NEFA could also induce formation of oxygen radicals that could quench $\mathrm{NO}$ and thus result in reduced $\mathrm{NO}$ action in vascular smooth muscle. However, short-term NEFA increases do not impair the blood flow response to endothelium independent vasodilator sodium nitroprusside. Because SNP acts as a direct donor of NO, this suggests similar NO availability and action under conditions of normal and short-term raised NEFA. Moreover, shortterm raised NEFA does not impair insulin-mediated vasodilation which is in large part NO dependent indicating intact endothelial NO production.

Short-term exposure to raised NEFA concentrations $(2 \mathrm{~h})$ causes impairment of endothelial function in lean normal insulin sensitive subjects and more prolonged exposure $(8 \mathrm{~h})$ is required to produce reduced rates of insulin-mediated glucose uptake (insulin resistance). If insulin-mediated vasodilation is tightly related to rates of insulin-mediated glucose uptake as previously suggested, it follows that NEFA increases which cause insulin resistance should also result in impaired insulin's vasodilatory action. To test this idea, healthy lean insulin-sensitive subjects were exposed to higher circulating NEFA concentrations both short term and long term. Short-term NEFA infusion superimposed on euglycaemic hyperinsulinaemia did not have any significant effect on insulin-mediated vasodilation, glucose uptake or NOx flux. In contrast, longer NEFA exposure resulted in a marked reduction of whole-body glucose uptake rates. Longer NEFA exposure not only caused a $35 \%$ fall in glucose uptake but also blunted insulin-mediated increases in LBF, and nearly completely abolished insulin-induced increases in NO flux. Thus, the results indicate that prolonged NEFA exposure results in concomitant and commensurate impairment of insulin-mediated vasodilation and insulin resistance. Stated differently, blunting of insulin's vascular action was observed only when a fall in insulin-mediated glucose uptake became evident. Importantly, throughout all groups, NEFA-induced changes in whole body glucose uptake correlated with NEFA-induced reductions in insulinmediated LBF strongly arguing in favour of a coupling of glucose metabolism and tissue perfusion (Fig. 8).

The results of our studies suggest that short-term NEFA exposure is sufficient to cause significant impairment of shear stress (basal NO dependent blood flow) and MCh-induced vasodilation. However, a much longer exposure to NEFA increases with concomitant induction of insulin resistance is required to cause impaired insulin-mediated vasodilation. The mechanism(s) for the different time courses required to affect endothelial responses to different stimuli are not clear. To better understand the mechanism(s) responsible for the difference in the time course effect of raised NEFA, one must consider that NO release accounts for only $50 \%$ of the vasodilation in response to $\mathrm{MCh}$, whereas insulin-mediated vasodilation depends almost exclusively on the production or release of $\mathrm{NO}$ or both. Other vasodilator mechanisms such as 


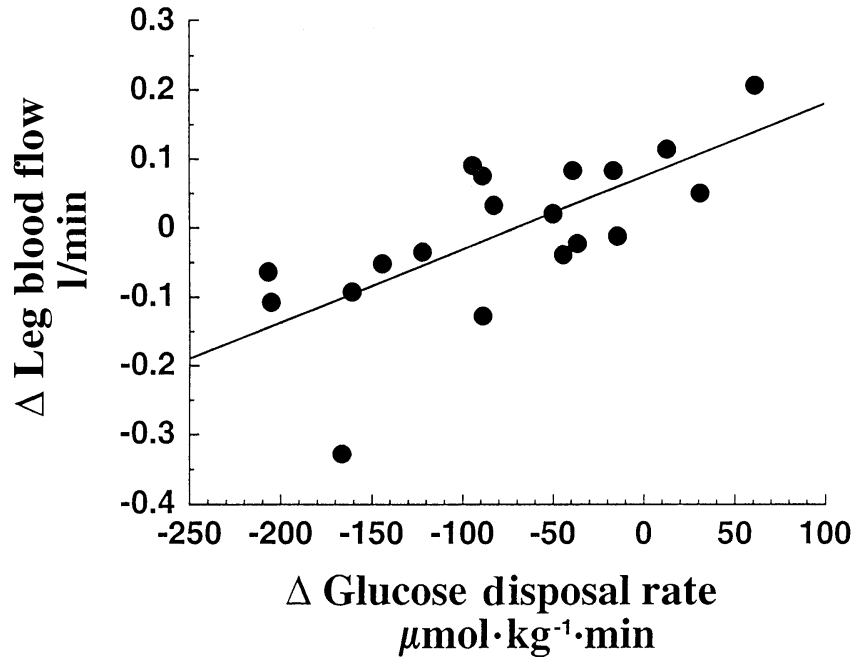

Fig. 8. Relation between non-esterified fatty acid induced changes in rates of leg blood flow ( $\Delta$ leg blood flow, $1 / \mathrm{min}$ ) and rates of whole body glucose uptake ( $\Delta$ glucose disposal rate) in response to raised non-esterified fatty acid superimposed on euglycaemic hyperinsulinaemia. $(r=0.706, p<0.001)$

endothelium dependent hyperpolarizing factor or cyclooxygenase products could contribute to the increases in shear stress-induced vasodilation. Therefore, impairment of non-NO-dependent vasodilator mechanisms by NEFA could be one explanation for the time course differences in response to NEFA to MCh and insulin.

On the other hand, the short-term NEFA increase inducing blunting of NO release during MCh stimulation with concomitant intact insulin-mediated vasodilation would suggest that short-term raised NEFA does not interfere with NO synthesis but could interfere with shear stress and muscarinic receptor signalling related to NOS activation. This interpretation is consistent with our data showing that short-term NEFA exposure causes decreased NO-dependent basal flow. Regardless of the signalling mechanisms by which NEFA elevation inhibits MCh-mediated vasodilation, it does not involve the rate-limiting step of $\mathrm{NO}$ generation because insulin stimulation induces vasodilation via by increased NO production even after 2-h and 4-h raised NEFA. In contrast to the effect of NEFA on MCh-induced vasodilation, the impaired insulin-mediated vasodilation required prolonged NEFA exposure and occurred concomitantly with the induction of insulin resistance. This suggests that NEFA induces insulin resistance simultaneously in skeletal muscle and vascular endothelium. In the skeletal muscle, the defect is reflected in reductions in glucose uptake whereas in the vascular endothelium it is reflected in reduced NO release.

The notion that raised NEFA impairs endothelial nitric oxide production is supported by studies in endothelial cell cultures. NEFA inhibits NO production in a dose-dependent fashion [75]. Because endothelial
NOS protein content was not altered by raised NEFA, they concluded that NEFA impaired NO production by decreasing endothelial NOS activity. One mechanism proposed is that the inhibition of eNOS is mediated by a PKC-dependent mechanism but studies have yielded conflicting results.

Raised NEFA has been shown to interfere with insulin signalling in skeletal muscle by reducing the activity of PI3K and this could represent the mechanism for NEFA-induced insulin resistance. Because insulin resistance is associated with reduced insulin-stimulated PI3K activation of vascular cells in vivo and in vitro as well as a reduction in its downstream signalling molecules including the serine and threonine kinase Akt, this set of findings suggests that shared insulin signalling pathways in skeletal muscle and vascular endothelium could be simultaneously affected by raised NEFA. Specifically, NEFA could impair PI3K both in skeletal muscle and in endothelium, thus causing impaired insulin-mediated glucose uptake and vasodilation respectively. This proposal is an explanation for the endothelial dysfunction observed in insulin-resistant states and, as such, provides a mechanism for the increased incidence of hypertension and macrovascular disease observed in these patients.

In summary, NEFA impairs both shear stress-mediated and insulin-mediated NO production. Impairment of shear stress-mediated NO production occurs after a relatively short time period of raised NEFA. In contrast, reductions in insulin-mediated vasodilation require prolonged exposure to raised NEFA and is only observed when rates of insulin-mediated glucose uptake are simultaneously reduced, indicating that metabolic coupling exists between insulin's effect to dilate skeletal muscle vasculature and its effect to promote glucose uptake in that tissue. The notion that vascular and metabolic effects of insulin could be coupled is supported by a strong correlation between NEFA-induced changes in glucose metabolism and blood flow increments (Fig. 8). Given that NEFA concentrations are constantly higher in insulin-resistant states of obesity and Type II diabetes, dysregulation of fatty acid metabolism could represent a shared and instrumental step leading to impairment of vascular reactivity and endothelial function and insulin-mediated glucose metabolism. The understanding of common and tissue specific effects of raised NEFA on insulin-receptor signalling events could identify targets for drug treatment that improve both glucose metabolism and vascular function. Improved vascular function should result in increased insulin sensitivity and decreased rates of macrovascular disease in these high-risk subjects.

Acknowledgements. This work was supported by grants DK42469, MO1-RR750-19, and DK20542 from the National Institutes of Health, and a Veterans Affairs Merit Review Award. The authors wish to thank J. Ballard for her expert and invaluable help in preparing the manuscript. 


\section{References}

1. Schulingkamp RJ, Pagano TC, Hung D, Raffa RB (2000) Insulin receptors and insulin action in the brain: review and clinical implications. Neurosci Biobehav Rev 24: 855-872

2. Freychet $P$ (2000) Insulin receptors and insulin actions in the nervous system. Diabetes Metab Res Rev 16: 390-392

3. Aspinwall CA, Lakey JR, Kennedy RT (1999) Insulinstimulated insulin secretion in single pancreatic beta cells. J Biol Chem 274: 6360-6365

4. Kulkarni RN, Bruning JC, Winnay JN, Postic C, Magnuson MA, Kahn CR (1999) Tissue-specific knockout of the insulin receptor in pancreatic beta cells creates an insulin secretory defect similar to that in type 2 diabetes. Cell 96: 329-339

5. Baron AD (1994) Hemodynamic actions of insulin. Am J Physiol 267: E187-E202

6. Kahn BB, Flier JS (2000) Obesity and insulin resistance. J Clin Invest 106: 473-481

7. Dunaif A (1993) Insulin resistance in polycystic ovarian syndrome. Ann N Y Acad Sci 687: 60-64

8. Laakso M (1996) Insulin resistance and coronary heart disease. Curr Opin Lipidol 7: 217-226

9. Fontbonne A, Charles MA, Thibult N et al. (1991) Hyperinsulinaemia as a predictor of coronary heart disease mortality in a healthy population: the Paris Prospective Study, 15-year follow-up. Diabetologia 34: 356-361

10. Fontbonne A, Eschwege E (1991) Insulin-resistance, hypertriglyceridaemia and cardiovascular risk: the Paris Prospective Study. Diabete Metab 17: 93-95

11. Liang C-S, Doherty JU, Faillace R et al. (1982) Insulin infusion in conscious dogs. Effects on systemic and coronary hemodynamics, regional blood flows, and plasma catecholamines. J Clin Invest 69: 1321-1336

12. Laakso M, Edelman SV, Brechtel G, Baron AD (1990) Decreased effect of insulin to stimulate skeletal muscle blood flow in obese men. J Clin Invest 85: 1844-1852

13. Anderson EA, Hoffman RP, Balon TW, Sinkey CA, Mark AL (1991) Hyperinsulinemia produces both sympathetic neural activation and vasodilation in normal humans. J Clin Invest 87: 2246-2252

14. Scherrer U, Randin D, Vollenweider P, Vollenweider L, Nicod P (1994) Nitric oxide release accounts for insulin's vascular effects in humans. J Clin Invest 94: 2511-2515

15. Yki-Jarvinen H, Young AA, Lamkin C, Foley JE (1987) Kinetics of glucose disposal in whole body and across the forearm in man. J Clin Invest 79: 1713-1719

16. Utriainen T, Malmstrom R, Makimattila S, Yki-Jarvinen H (1993) Methodological aspects, dose-response characteristics and causes of interindividual variation in insulin stimulation of limb blood flow in normal subjects. Diabetologia 38: 555-564

17. Baron AD, Brechtel G (1993) Insulin differentially regulates systemic and skeletal muscle vascular resistance. Am J Physiol 265: E61-E67

18. Mather K, Laakso M, Edelman S, Hook G, Baron A (2000) Evidence for physiological coupling of insulin-mediated glucose metabolism and limb blood flow. Am J Physiol Endocrinol Metab 279: E1264-E1270

19. Baron AD, Brechtel-Hook G, Johnson A, Cronin J, Leaming R, Steinberg HO (1996) Effect of perfusion rate on the time course of insulin mediated skeletal muscle glucose uptake. Am J Physiol 271: E1067-E1072

20. Jamerson KA, Nesbitt SD, Amerena JV, Grant E, Julius S (1996) Angiotensin mediates forearm glucose uptake by hemodynamic rather than direct effects. Hypertension 27: $854-858$
21. Jamerson KA, Smith SD, Amerena JV, Grant E, Julius S (1994) Vasoconstriction with norepinephrine causes less forearm insulin resistance than a reflex sympathetic vasoconstriction. Hypertension 23: 1006-1011

22. Ferrannini E, Taddei S, Santoro D et al. (1998)Independent stimulation of glucose metabolism and $\mathrm{Na}^{+}-\mathrm{K}^{+}$exchange by insulin in the human forearm. Am J Physiol 255: E953-E958

23. Baron AD, Tarshoby M, Hook G et al. (2000) Interaction between insulin sensitivity and muscle perfusion on glucose uptake in human skeletal muscle: evidence for capillary recruitment. Diabetes 49: 768-774

24. Natali A, Qjinones A, Pecori N, Sanna G, Toschi E, Ferrannini E (1998) Vasodilation with sodium nitroprusside does not improve insulin action in essential hypertension. Hypertension 31: 632-636

25. Natali A, Bonadonna R, Santoro D et al. (1994) Insulin resistance and vasodilation in essential hypertension. Studies with adenosine. J Clin Invest 94: 1570-1576

26. Nuutila P, Raitakari M, Laine H et al. (1996) Role of blood flow in regulating insulin-stimulated glucose uptake in humans. Studies using bradykinin, [15O]water, and [18F]fluoro-deoxy-glucose and positron emission tomography $\mathrm{J}$ Clin Invest 97: 1741-1747

27. Clark AD, Barrett EJ, Rattigan S, Wallis MG, Clark MG (2001) Insulin stimulates laser Doppler signal by rat muscle in vivo, consistent with nutritive flow recruitment. Clin Sci (Colch) 100: 283-290

28. Youd JM, Rattigan S, Clark MG (2000) Acute impairment of insulin-mediated capillary recruitment and glucose uptake in rat skeletal muscle in vivo by TNF-alpha. Diabetes 49: 1904-1909

29. Rattigan S, Clark MG, Barrett EJ (1997) Hemodynamic actions of insulin in rat skeletal muscle. Evidence for capillary recruitment. Diabetes 46: 1381-1388

30. Rattigan S, Clark MG, Barrett EJ (1999) Acute vasoconstriction-induced insulin resistance in rat muscle in vivo. Diabetes 48: 564-569

31. Yki-Jarvinen H, Utriainen T (1998) Insulin-induced vasodilation: physiology or pharmacology? Diabetologia 41: 369-379

32. Steinberg HO, Baron AD (1999) Insulin-mediated vasodilation: why one's physiology could be the other's pharmacology. Diabetologia 42: 493-495

33. Furchgott RF, Zawadzki JV (1980) The obligatory role of endothelial cells in the relaxation of arterial smooth muscle by acetylcholine. Nature 288: 373-382

34. Moncada S, Palmer RMJ, Higgs EA (1988) The discovery of nitric oxide as the endogenous vasodilator. Hypertension 12: $365-372$

35. Ignarro LJ (1990) Nitric Oxide - A novel signal transduction mechanism for transcellular communication. Hypertension 16: 477-483

36. Vallance P, Collier J, Moncada S (1989) Effects of endothelium-derived nitric oxide on peripheral arteriolar tone in man. Lancet 2: 997-1000

37. Steinberg HO, Brechtel G, Johnson A, Fineberg N, Baron AD (1994) Insulin-mediated skeletal muscle vasodilation is nitric oxide dependent. J Clin Invest 94: 1172-1179

38. Vollenweider P, Tappy L, Randin D et al. (1993) Differential effects of hyperinsulinemia and carbohydrate metabolism on sympathetic nerve activity and muscle blood flow in humans. J Clin Invest 92: 147-154

39. Tack CJ, Smits P, Willemsen JJ, Lenders JW, Thien T, Lutterman JA (1996) Effects of insulin on vascular tone and sympathetic nervous system in NIDDM. Diabetes 45: $15-22$ 
40. Cardillo C, Nambi SS, Kilcoyne CM et a. (1999) Insulin stimulates both endothelin and nitric oxide activity in the human forearm. Circulation 100: 820-825

41. Laight DW, Kaw AV, Carrier MJ, Anggard EE Pharmacological modulation of endothelial function by insulin in the rat aorta. J Pharm Pharmacol 50: 1117-1120

42. Cipolla MJ, Harker CT, Porter JM (1996) Endothelial function and adrenergic reactivity in human type-II diabetic resistance arteries. J Vasc Surg 23: 940-949

43. Touyz RM, Tolloczko B, Schiffrin EL (1994) Insulin attenuates agonist-evoked calcium transients in vascular smooth muscle cells. Hypertension 23[Suppl 1]: I25-128

44. Kim YC, Zemel MB (1993) Insulin increases vascular smooth muscle recovery from intracellular calcium loads. Hypertension 22: 74-77

45. Zeng G, Quon MJ (1996) Insulin stimulated production of nitric oxide is inhibited by Wortmannin. Direct measurement in vascular endothelial cells J Clin Invest 98: 894-898

46. Zeng G, Nystrom FH, Ravichandran LV et al. (2000) Roles for insulin receptor, PI3-kinase, and Akt in insulinsignaling pathways related to production of nitric oxide in human vascular endothelial cells. Circulation 101: 15391545

47. Dimmeler S, Fleming I, Fisslthaler B, Hermann C, Busse R, Zeiher AM (1999) Activation of nitric oxide synthase in endothelial cells by Akt- dependent phosphorylation. Nature 399: 601-605

48. Fulton D, Gratton JP, McCabe TJ et al. (1999) Regulation of endothelium-derived nitric oxide production by the protein kinase Akt. Nature 399: 597-601

49. Kuboki K, Jiang ZY, Takahara N et al. (2000) Regulation of endothelial constitutive nitric oxide synthase gene expression in endothelial cells and in vivo: a specific vascular action of insulin. Circulation 101: 676-681

50. Laakso M, Edelman SV, Brechtel G, Baron AD (1992) Impaired insulin-mediated skeletal muscle blood flow in patients with NIDDM. Diabetes 41: 1076-1083

51. Steinberg HO, Cressman E, Wu Y et al. (1997) Insulin mediated nitric oxide production is impaired in insulin resistance. Diabetes 46[Suppl 1]: 24A

52. Vita JA, Treasure CB, Nabel EG et al. (1990) Coronary vasomotor responses to acetylcholine relates to risk factors for coronary artery disease. Circulation 81: 491-497

53. Schachinger V, Britten MB, Zeiher AM (2000) Prognostic impact of coronary vasodilator dysfunction on adverse long- term outcome of coronary heart disease. Circulation 101: 1899-1906

54. Suwaidi JA, Hamasaki S, Higano ST, Nishimura RA, Holmes DR Jr, Lerman A (2000) Long-term follow-up of patients with mild coronary artery disease and endothelial dysfunction. Circulation 101: 948-954

55. Selwyn AP, Kinlay S, Creager M, Libby P, Ganz P (1997) Cell dysfunction in atherosclerosis and the ischemic manifestations of coronary artery disease. Am J Cardiol 79: 17-23

56. Manson JE, Willett WC, Stampfer MJ et al. (1995) Hennekens $\mathrm{CH}$, Speizer FE. Body weight and mortality among women. N Eng J Med 333: 677-685

57. Haffner SM, Lehto S, Ronnemaa T, Pyorala K, Laakso M (1998) Mortality from coronary heart disease in subjects with type 2 diabetes and in nondiabetic subjects with and without prior myocardial infarction. N Engl J Med 339: 229-234
58. Adlerberth AM, Rosengren A, Wilhelmsen L (1998) Diabetes and long-term risk of mortality from coronary and other causes in middle-aged Swedish men. A general population study. Diabetes Care 21: 539-545

59. Laakso M, Kuusisto J (1996) Epidemiological evidence for the association of hyperglycaemia and atherosclerotic vascular disease in non-insulin-dependent diabetes mellitus. Ann Med 28: 415-418

60. Steinberg HO, Paradisi G, Cronin J et al. (2000) Type II diabetes abrogates sex differences in endothelial function in premenopausal women. Circulation 101: 2040-2046

61. Hattersley AT, Tooke JE (1999) The fetal insulin hypothesis: an alternative explanation of the association of low birthweight with diabetes and vascular disease. Lancet 353: 1789-1792

62. Jiang BH, Zheng JZ, Aoki M, Vogt PK (2000) Phosphatidylinositol 3-kinase signaling mediates angiogenesis and expression of vascular endothelial growth factor in endothelial cells. Proc Natl Acad Sci USA 97: 1749-1753

63. O'Brien SF, Russell JC, Dolphin PJ, Davidge ST (2000) Vascular wall function in insulin-resistant JCR:LA-cp rats: role of male and female sex. J Cardiovasc Pharmacol 36: 176-181

64. Jiang ZY, Lin YW, Clemont A et al. (1999) Characterization of selective resistance to insulin signaling in the vasculature of obese Zucker (fa/fa) rats. J Clin Invest 104: 447-457

65. Groop LC, Saloranta C, Shank M, Bonadonna RC, Ferrannini E, DeFronzo RA (1991) The role of free fatty acid metabolism in the pathogenesis of insulin resistance in obesity and noninsulin-dependent diabetes mellitus. J Clin Endocrinol Metab 72: 96-107

66. Boden G (1996) Fatty acids and insulin resistance. Diabetes Care 19: 394-395

67. Roden M, Price TB, Perseghin G et al. (1996) Mechanism of free fatty acid-induced insulin resistance in humans. J Clin Invest 97: 2859-2865

68. Gumbiner B, Mucha JF, Lindstrom JE, Rekhi I, Livingston JN (1996) Differential effects of acute hypertriglyceridemia on insulin action and insulin receptor autophosphorylation. Am J Physiol 270: E424-E429

69. Roden DM (1993) Pharmcokinetics of amiodarone: Implications for drug therapy. Am J Cardiol 72: 45F-50F

70. Steinberg HO, Paradisi G, Hook G, Crowder K, Cronin J, Baron AD (2000) Free fatty acid elevation impairs insulinmediated vasodilation and nitric oxide production. Diabetes 49: 1231-1238

71. Dresner A, Laurent D, Marcucci M ET AL. (1999) Effects of free fatty acids on glucose transport and IRS-1-associated phosphatidylinositol 3-kinase activity. J Clin Invest 103: 253-259

72. Steinberg HO, Tarshoby M, Monestel R et al. (1997) Elevated circulating free fatty acid levels impair endotheliumdependent vasodilation. J Clin Invest 100: 1230-1239

73. de Kreutzenberg SV, Crepaldi C, Marchetto S et al. (2000) Plasma free fatty acids and endothelium-dependent vasodilation: effect of chain-length and cyclooxygenase inhibition. J Clin Endocrinol Metab 85: 793-798

74. Lundman P, Eriksson M, Schenck-Gustafsson K, Karpe F, Tornvall P (1997) Transient triglyceridemia decreases vascular reactivity in young, healthy men without risk factors for coronary heart disease. Circulation 96: 3266-3268

75. Gupta MP, Steinberg H, Baron A, Hart CM (1998) Fatty acids impair nitric oxide production in cultured endothelial cells. J Investig Med 46: 288A 